\title{
Caratheodory operator of differential forms
}

\author{
Zhaoyang Tang ${ }^{*}$ and Jianmin Zhu
}

* Correspondence: tzymath@gmail. com

Department of Mathematics and System Science, National University of Defense Technology, Changsha, PR China

\begin{abstract}
This article is devoted to extensions of some existing results about the Caratheodory operator from the function sense to the differential form situation. Similarly as the function sense, we obtain the convergence of sequences of differential forms defined by the Caratheodory operator. The main result in this article is the continuity and mapping property from one space of differential forms to another under some dominated conditions.
\end{abstract}

Keywords: differential forms, Caratheodory operator, continuity of operator

\section{Introduction}

It is well known that differential forms are generalizations of differentiable functions in $R^{N}$ and have been applied to many fields, such as potential theory, partial differential equations, quasi-conformal mappings, nonlinear analysis, electromagnetism, and control theory [1-12].

One of the important work in the field of differential forms is to develop various kinds of estimates and inequalities for differential forms under some conditions. These results have wide applications in the A-harmonic equation, which implies more versions of harmonic equations for functions $[1,5,6]$.

The Caratheodory operator arose from the extension of Peano theorem about the existence of solutions to a first-order ordinary differential equation, which says that this kind equation has a solution under relatively mild conditions. It is very interesting to characterize equivalently the Caratheodory's conditions and the continuity of Caratheodory operator, which form classic examples to discuss boundedness and continuity of nonlinear operators and play an important part in advanced functional analysis.

For general function space, we define the Caratheodory operator as in $[13,14]$.

Definition 1.1. Suppose that $G$ is measurable in $R^{N}$, and $0<m e s G \leq+\infty$. We say that function $f(x, \omega)(x \in G,-\infty<\omega<+\infty)$ satisfies the Caratheodory conditions, if

1. for almost all $x \in G, f(x, \omega)$ is continuous with respect to $\omega$; and

2. for any $\omega, f(x, \omega)$ is measurable about $x$ on $G$.

For the function $f(x, \omega)$ with Caratheodory conditions, we define the Caratheodory operator $T: G \times R \rightarrow R$ by

$$
T \omega(x)=f(x, \omega(x)) .
$$

There are some essential results for Caratheodory operator as follows.

\section{Springer}

(c) 2011 Tang and Zhu; licensee Springer. This is an Open Access article distributed under the terms of the Creative Commons Attribution License (http://creativecommons.org/licenses/by/2.0), which permits unrestricted use, distribution, and reproduction in any medium, provided the original work is properly cited. 
Lemma 1.1. Suppose that mes $\Omega<+\infty$. Then $f(x, \omega)$ satisfies the Caratheodory condition if and only if for any $\eta>0$, there exists a bounded open set $F \subset \Omega$ with mesF $>m e s \Omega-\eta$, such that $f(x, \omega)$ is continuous on $F \times R$.

Lemma 1.2. Suppose that mes $\Omega<\infty$. If $\omega_{n}(s)(n=1,2, \ldots)$ convergence in measure to $\omega(s)$ on $\Omega$, then $T \omega_{n}(s)=f\left(s, \omega_{n}(s)\right)(n=1,2, \ldots)$ convergence in measure to $T \omega(s)$ on $\Omega$.

Theorem 1.1. The Caratheodory operator $T$ maps $L^{p_{1}}(\Omega)$ into $L^{p_{2}}(\Omega)$ if and only if there exists a real number $b>0$, and a function $a(x) \geq 0, a(x) \in L^{p_{2}}(\Omega)$ satisfies the following inequality

$$
|f(x, \omega)| \leq a(x)+b|\omega|^{\frac{p_{1}}{p_{2}}} \quad(x \in \Omega, \omega \in(-\infty,+\infty)) .
$$

This article is to extend the above results to the space of differential forms.

\section{Some preliminaries about differential forms}

First, we introduce some notations and preliminaries about differential forms. Let $\Omega$ denote an open subset of $R^{N}, N \geq 2$ and $R=R^{1}$, and the $n$-dimensional Lebesgue measure of a set $\Omega \subset R^{N}$ is denoted by $\operatorname{mes}(\Omega)$. Let $\left\{e_{1}, e_{2}, \ldots, e_{n}\right\}$ denote the standard orthogonal basis of $R^{N} . \Lambda^{l}\left(R^{N}\right)$ is the linear space of l-covectors, generated by the exterior products $e_{I}=e_{i_{1}} \wedge e_{i_{2}} \wedge \cdots \wedge e_{i_{v}}$, corresponding to all ordered $l$-tuples $I=\left(i_{1}\right.$, $\left.i_{2}, \ldots, i_{l}\right), \quad 1 \leq i_{1}<i_{2}<\ldots<i_{l} \leq N, l=0,1, \ldots, N$. The Grassman algebra $\wedge=\wedge\left(R^{N}\right)=\oplus_{l=0}^{N} \wedge^{\imath}$ is a graded algebra with respect to the exterior products.

A differential $l$-form $\omega$ on $\Omega$ is a Schwartz distribution on $\Omega$ with values in $\Lambda\left(R^{N}\right)$. Let $D^{\prime}\left(\Omega, \Lambda^{l}\right)$ denote the space of all differential $l$-forms, and $L^{p}\left(\Omega, \Lambda^{l}\right)$ denote the space composed by the $l$-forms

$$
\omega(x)=\sum_{I} \omega_{I}(x) d x_{I}=\sum_{I} \omega_{i_{1} i_{2} \ldots i_{\iota}}(x) d x_{i_{1}} \wedge d x_{i_{2}} \wedge \cdots \wedge d x_{i_{\iota^{\prime}}}
$$

where $\omega_{I} \in L^{p}(\Omega, R)$ for all ordered $l$-tuples $I$. Then $L^{p}\left(\Omega, \Lambda^{l}\right), p \geq 1$ is a Banach space with norm

$$
\|\omega\|_{p, \Omega}=\left(\int \Omega|\omega(x)|^{p} d x\right)^{1 / p}=\left(\int \Omega\left(\sum_{I}\left|\omega_{I}(x)\right|^{2}\right)^{p / 2} d x\right)^{1 / p}<+\infty .
$$

We see that

$$
\left\{d x_{i_{1}} \wedge d x_{i_{2}} \wedge \ldots \wedge d x_{i_{\iota}}, 1 \leq i_{1}<i_{2}<\cdots<i_{\iota} \leq N\right\}
$$

is a basis of the space $\wedge^{l}$, then $\operatorname{dim}\left(\wedge^{l}\right)=\operatorname{dim}\left(\wedge^{l}\left(R^{N}\right)\right)=\sum_{l=0}^{N} C_{N}^{l}$ and

$$
\operatorname{dim}(\wedge)=\sum_{l=0}^{N} \operatorname{dim}\left(\wedge^{l}\left(R^{N}\right)\right)=\sum_{l=0}^{N} C_{N}^{l}=2^{N}
$$

Then we define the Caratheodory conditions and Caratheodory operator for differential forms.

Definition 2.1. For a mapping $f: \Omega \times \Lambda^{l} \rightarrow \Lambda^{l}$, where $\Omega$ is an open set in $R^{N}$, we say that $f$ satisfies Caratheodory conditions if 1. for all most $s \in \Omega, f(s, \omega)$ is continuous with respect to $\omega$, which means that $f$ can be expanded as $f(s, \omega)=\Sigma_{J} f_{J}(s, \omega) d x_{J}$ where 
$f_{J}: \Omega \times \wedge^{l} \rightarrow R$ and $f_{J}(s, \omega)$ is continuous about $\omega$ for all most $s \in \Omega$; and 2 . for any fixed $\omega=\Sigma_{I} \omega d x_{I} \in \Lambda^{l}, f(s, \omega)$ is measurable about s, which means that each coefficient function $f_{\mathcal{J}}(s, \omega)$ is measurable about $s$ for any fixed $\omega \in \Lambda^{l}$.

Throughout this article we assume that $f(s, \omega)$ satisfies the Caratheodory condition (C-condition). Similarly, we can define the continuity of $f(s, \omega)$ about $(s, \omega) \in \Omega \times \Lambda^{l}$.

Definition 2.2. Suppose that $\Omega \subset R^{N}$ is measurable $\operatorname{set}(0<$ mes $\Omega \leq+\infty)$, and $f: \Omega \times$ $\Lambda^{l} \rightarrow \Lambda^{l}$. We define the Caratheodory operator $T: \Lambda^{l} \rightarrow \Lambda^{l}$ for differential forms by

$$
T \omega(s)=f(s, \omega(s)) .
$$

\section{Main results and proofs}

There is a necessary and sufficient condition of the Caratheodory conditions:

Lemma 3.1. Suppose that mes $\Omega<+\infty$. Then $f(x, \omega)$ satisfies the Caratheodory condition, if and only if, for any $\eta>0$, there exists a bounded closed set $F \subset \Omega$, with mes $F$ $>m e s \Omega-\eta$, such that $f(x, \omega)$ is continuous on $F \times \wedge^{l}$.

Proof. Proof of sufficiency.

According to the hypothesis, there exists a bounded open set $F_{n} \subset \Omega$, with $m e s F_{n}>$ mes $\Omega-\frac{1}{n} \quad(n=1,2, \ldots)$, such that $f(x, \omega)$ is continuous on $F_{n} \times \Lambda^{l}$. Let $F=\cup_{m=1}^{+\infty} F_{n} \subset \Omega$, then mesF $=$ mes $\Omega$, and when $x \in F, f(x, \omega)$ is continuous on $\Lambda^{l}$. Hence, the first one of Caratheodory conditions is satisfied. For fixed $\omega \in \Lambda^{l},\left\{x \in F_{n} \mid f_{I}\right.$ $(x, \omega) \geq a\}(a \in R)$ is bounded and closed. Then

$$
\left\{x \in F \mid f_{I}(x, \omega) \geq a\right\}=\bigcup_{n=1}^{+\infty}\left\{x \in F_{n} \mid f_{I}(x, \omega) \geq a\right\}
$$

is measurable, so that $f(x, \omega)=\sum_{I} f(x, \omega) d x_{I}$ is measurable on $F$ respect to $x$, then it is measurable on $\Omega$. Hence, the second one of Caratheodory conditions is satisfied.

Proof of necessity.

For a given $\eta>0$, we only need to prove the following result: there exists a bounded closed set $F_{n} \subset \Omega$ with $\operatorname{mes} F_{n}>\operatorname{mes} \Omega-\frac{\eta}{2^{n}}$ and $\delta_{n}>0(n=1,2, \ldots)$ such that for any $x_{1}, x_{2} \in F_{n}$ with $\operatorname{dist}\left(x_{1}, x_{2}\right)<\delta_{n}, \omega_{1, I}, \omega_{2, I} \in[-n, n],\left|\omega_{1, I}-\omega_{2, I}\right|<\delta_{n}$, where $\omega_{i}=\sum_{I} \omega_{i}$, ${ }_{I} d x_{i}, \omega_{i, I} \in R, i=1$, 2, we have

$$
\left|f\left(x_{1}, \omega_{1}\right)-f\left(x_{2}, \omega_{2}\right)\right|<\frac{1}{n} \quad(n=1,2, \ldots) .
$$

Actually, if we have proved this conclusion, then $F=\bigcap_{n=1}^{+\infty} F_{n} \subset \Omega$ is bounded and closed and satisfies

$$
\operatorname{mes}(\Omega \backslash F)=\operatorname{mes}\left(\bigcup_{n=1}^{+\infty}\left(\Omega \backslash F_{n}\right) \leq \sum_{n=1}^{+\infty} \operatorname{mes}\left(\Omega \backslash F_{n}\right)<\sum_{n=1}^{+\infty} \frac{\eta}{2^{n}}=\eta .\right.
$$

Then we can prove $f(x, \omega)$ is continuous on $F \times \wedge^{l}$ as follows. For any given $(x, \omega) \in$ $F \times \Lambda^{l}$, and $\varepsilon>0$, we select $n_{0}$ with $\frac{1}{n_{0}}<\varepsilon$ and $\left|\omega_{1, I}\right|<n_{0}-1$ for all $I$. When $\left(x_{2}, \omega_{2}\right)$ in $F \quad \times \quad \Lambda^{l}$ satisfies $\operatorname{dist}\left(x_{1}, x_{2}\right)<\delta=\min \left\{\delta_{n_{0}}, 1\right\},\left|\omega_{1, I}-\omega_{2, I}\right|<\delta$, we have $x_{1}, x_{2} \in F \subset F_{n_{0}}, \operatorname{dist}\left(x_{1}, x_{2}\right)<\delta_{n_{0}},\left|\omega_{2, I}\right|<\delta+n_{0}-1 \leq n_{0}$, and 


$$
\left|f\left(x_{1}, \omega_{1}\right)-f\left(x_{2}, \omega_{2}\right)\right|<\frac{1}{n_{0}}<\varepsilon .
$$

Thus, $f(x, \omega)$ is continuous on $F \times \wedge^{l}$. Set

$$
\Omega_{0}=\left\{x \in \Omega \mid f(x, \omega) \text { is continuous on }-\infty<\omega_{I}<+\infty \text { as function about } \omega\right\} .
$$

According to the first one of Caratheodory conditions, we have mes $\Omega_{0}=$ mes $\Omega$. Let

$$
\begin{aligned}
\Omega_{m, n}= & \left\{x \in \Omega_{0} \mid \omega_{1, I}, \omega_{2, I} \in[-n, n], \text { and for all } I\left|\omega_{1, I}-\omega_{2, I}\right|<\frac{1}{m}\right. \\
& \text { contains that } \left.\left|f\left(x, \omega_{1}\right)-f\left(x, \omega_{2}\right)\right| \leq \frac{1}{3 n}\right\} \quad(n=1,2, \ldots) .
\end{aligned}
$$

With the density of the rational number, we have $\Omega_{0} \backslash \Omega_{m, n}=\left\{x \in \Omega_{0} \mid\right.$ there exists $\omega_{1, I}, \quad \omega_{2, I} \quad \in \quad\left[\begin{array}{lll}-n, & n], & \text { such }\end{array}\right.$ $\left.\left|\omega_{1, I}-\omega_{2, I}\right|<\frac{1}{m},\left|f\left(x, \omega_{1}\right)-f\left(x, \omega_{2}\right)\right|>\frac{1}{3 n}\right\}=\left\{x \in \Omega_{0} \mid\right.$ there exists rational number $\omega_{i, I}$ $\in[-n, n], i=1,2$, such that $\left.\left|\omega_{1, I}-\omega_{2, I}\right|<\frac{1}{m},\left|f\left(x, \omega_{1}\right)-f\left(x, \omega_{2}\right)\right|>\frac{1}{3 n}\right\}$.

With the second one of Caratheodory condition, for fixed $\omega_{1}, \omega_{2},\left\{x \in \Omega_{0}|| f\left(x, \omega_{1}\right)-f\left(x, \omega_{2}\right) \mid>\frac{1}{3 n}\right\}$ is measurable. Thus $\Omega_{0} \backslash \Omega_{m, n}$ (as the countable union of such able sets) is measurable, too. So $\Omega_{m, n}$ is measurable. Obviously, for fixed $n$ we have $\Omega_{1, n} \subset \Omega_{2, n} \subset \ldots$. Let $E_{n}=\bigcup_{m=1}^{+\infty} \Omega_{m, n} \subset \Omega_{0}$. We will prove that $E_{n}=\Omega_{0}$. Actually, if $E_{n} \neq \Omega_{0}$, then there exists $x_{0} \in \Omega_{0} \backslash E_{n}=\bigcap_{m=1}^{+\infty}\left(\Omega_{0} \backslash \Omega_{m, n}\right)$. Thus, there exist $\omega_{i}^{(m)}=\sum_{I} \omega_{i, I}^{(m)} d x_{I}, i=1,2$ with $\omega_{1, I}^{(m)}, \omega_{2, I}^{(m)} \in[-n, n]$, such that

$$
\left|\omega_{1, I}^{(m)}-\omega_{2, I}^{(m)}\right|<\frac{1}{m}
$$

and

$$
\left|f\left(x_{0}, \omega_{1}^{(m)}\right)-f\left(x_{0}, \omega_{2}^{(m)}\right)\right|>\frac{1}{3 n}(m=1,2, \ldots) .
$$

This obviously contradicts the uniform continuity of function $f\left(x_{0}, \omega\right)$, where $\omega=\Sigma_{I}$ $\omega_{I} d x_{I}$ with $-n \leq \omega_{I} \leq n$ for any $I$. Hence, we have proved $E_{n}=\Omega_{0}(n=1,2, \ldots)$.

Then we have

$$
\lim _{m \rightarrow+\infty} \operatorname{mes} \Omega_{m, n}=m e s \Omega_{0} \quad(n=1,2, \ldots) .
$$

For given $n$, we select $m_{0}$ such that

$$
m e s \Omega_{m_{0}, n}>m e s \Omega_{0}-\frac{1}{2^{n}} \frac{\eta}{3} \text {. }
$$

For any $I$, we divide interval $[-n, n]$ into $s=2 n m_{0}$ subintervals, and the endpoints of these subintervals are

$$
-n=\omega_{I}^{(0)}<\omega_{I}^{(1)}<\omega_{I}^{(2)}<\cdots<\omega_{I}^{(s)}=n,
$$

where $I=\left(i_{1}, i_{2}, \ldots, i_{l}\right)$ and the number of all $I$ is $t=C_{n}^{l}$. Using Luzin theorem, there exist bounded closed sets $D_{j} \subset \Omega_{0}$, such that 


$$
m e s D_{j}>\operatorname{mes} \Omega_{0}-\frac{\eta}{3(s+1)^{N} 2^{n}}
$$

and $f_{I}\left(x, \omega^{(j)}\right)$ is continuous on $D_{j}$ with respect to $x$ (thereby uniform continuous), where $\omega^{(j)}=\sum_{I} \omega^{(S I)} d x_{I}$ stands for $\omega_{I}^{(S I)}$ selected from $\omega_{I}^{(0)}, \omega_{I}^{(1)}, \ldots, \omega_{I}^{(s)}$ for different $I$. Then $\omega^{(j)}=\sum_{I} \omega_{I}^{(S I)} d x_{I} \in \wedge^{l}$, and we know the total amount of these $\omega^{(j)} S$ is $(s+1)^{N} \triangleq r$.

Let $D=\bigcap_{j=1}^{r} D_{j}$. According to the uniform continuity, there exists $\delta>0$ such that

$$
\left|f\left(x_{1}, \omega^{(j)}\right)-f\left(x_{2}, \omega^{(j)}\right)\right|<\frac{1}{3 n}, \text { for all } x_{1}, x_{2} \in D
$$

and

$$
\operatorname{dist}\left(x_{1}, x_{2}\right)<\delta \text { for } j=1,2, \ldots, t .
$$

Now we select closed set $F_{n} \subset \Omega_{m 0, n} \cap D$ such that

$$
\operatorname{mes} F_{n}>\operatorname{mes}\left(\Omega_{m_{0}, n} \cap D\right)-\frac{1}{2^{n}} \frac{\eta}{3},
$$

and $\delta_{n}$ satisfies $0<\delta_{n}<\min \left\{\delta, \frac{1}{m_{0}}\right\}$. We shall prove that $F_{n}$ and $\delta_{n}$ are those that we need.

Actually,

$$
\begin{aligned}
\Omega_{0} \backslash\left(\Omega_{m_{0}, n} \cap D\right) & =\left(\Omega \backslash \Omega_{m_{0}, n}\right) \cup\left(\Omega_{0} \backslash D\right) \\
& =\left(\Omega \backslash \Omega_{m_{0}, n}\right) \cup\left(\bigcup_{j=1}^{r}\left(\Omega_{0} \backslash D_{i}\right)\right),
\end{aligned}
$$

then we have

$$
\begin{aligned}
\operatorname{mes}\left(\Omega_{0} \backslash\left(\Omega_{m_{0}, n} \cap D\right)\right) & \leq \operatorname{mes}\left(\Omega_{0} \backslash \Omega_{m_{0}, n}\right)+\sum_{j=1}^{r} \operatorname{mes}\left(\Omega_{0} \backslash D_{j}\right) \\
& <\frac{1}{2^{n}} \frac{\eta}{3}+\sum_{j=1}^{r} \frac{\eta}{3 r 2^{n}}=\frac{1}{2^{n}} \frac{2 \eta}{3} .
\end{aligned}
$$

This leads to

$$
\operatorname{mes}\left(\Omega_{m_{0}, n} \cap D\right)>\operatorname{mes} \Omega_{0}-\frac{1}{2^{n}} \frac{2 \eta}{3} .
$$

Thus,

$$
m e s F_{n}>m e s \Omega_{0}-\frac{\eta}{2^{n}}=m e s \Omega-\frac{\eta}{2^{n}} .
$$

Suppose that $x_{1}, x_{2} \in F_{n}, d\left(x_{1}, x_{2}\right)<\delta_{n}, \omega_{i, I} \in[-n, n](i=1,2)\left|\omega_{1, I}-\omega_{2, I}\right|<\delta_{n}$, and $\omega_{I}^{(i+1)}-\omega_{I}^{(i)}=\frac{1}{m_{0}}\left(\delta_{n}<\frac{1}{m_{0}}\right), i=0,1,2, \ldots, s-1, \quad$ and there exist some $\omega^{(j)}=\sum_{I} \omega_{I}^{(S I)} d x_{I}$ such that for any $I$, we have $\left|\omega_{1, I}-\omega_{2, I}^{(S I)}\right|<\frac{1}{m_{0}}$. Then, we obtain 


$$
\begin{aligned}
\left|f\left(x_{1}, \omega_{1}\right)-f\left(x_{1}, \omega_{2}\right)\right| & \leq\left|f\left(x_{2}, \omega_{2}\right)-f\left(x_{2}, \omega^{(j)}\right)\right|+\left|f\left(x_{2}, \omega^{(j)}\right)-f\left(x_{1}, \omega^{(j)}\right)\right| \\
& +\left|f\left(x_{1}, \omega^{(j)}\right)-f\left(x_{1}, \omega_{1}\right)\right| \\
& \leq \frac{1}{3 n}+\frac{1}{3 n}+\frac{1}{3 n}=\frac{1}{n} .
\end{aligned}
$$

The proof of necessity is finished.

Actually, [15] gave a proof of the condition that the $u$ in $f(x, u)$ is a normal $l$-dimensional vector. With this lemma, we have the following result.

Lemma 3.2. Suppose that mes $\Omega<+\infty$. If for $\omega(x)=\Sigma_{I} \omega_{I}(x) d x_{I}, \omega_{I}(x)$ is measurable on $\Omega$, then $T \omega(x)=f(x, \omega(x))$ is measurable on $\Omega$.

Proof. According to Lemma 3.1, there exists a closed set $F_{n} \subset \Omega$ with $m e s F_{n}>\operatorname{mes} \Omega-\frac{1}{n} \quad(n=1,2, \ldots)$, such that $f(x, \omega)$ is continuous on $F_{n} \times \wedge^{l}$. Suppose that $F_{n} \subset F_{n+1}(n=1,2, \ldots)$, otherwise let $\bigcup_{k=1}^{n} F_{k}$ be the new $F_{n}$. From Luzin Theorem, there exists a closed set $D_{n, I} \subset F_{n}$, mes $D_{n, I}>\operatorname{mes} F_{n}-\frac{1}{n t}\left(t=C_{N}^{l}\right)$ such that $\omega_{I}(x)$ is continuous on $D_{n, I}$. Similarly, supposing $D_{n, I} \subset D_{n+1, I}(n=1,2, \ldots)$, and $D_{n}=\cap_{I} D_{n, I} \subset$ $\mathrm{F}_{n}$, we have $\operatorname{mes}\left(F_{n} \backslash D_{n}\right) \leq \sum_{I}\left(m e s F_{n}-\operatorname{mes} D_{n, I}\right)=\sum_{I} \frac{1}{n t}=\frac{1}{n}$, which deduces that for any $I, \omega_{I}(x)$ is continuous on $D_{n}$. We suppose $D_{n} \subset D_{n+1}(n=1,2, \ldots)$ just as $F_{n}$. Let $D=\bigcup_{n=1}^{\infty} D_{n,} H=\Omega \backslash D$, then

$$
m e s D=\lim _{n \rightarrow \infty} m e s D_{n}=m e s \Omega \text {. }
$$

Hence, we have $m e s H=0$. For any rational number $a$,

$$
\left\{x \mid x \in D_{n}, f_{I}(x, \omega(x)) \geq a\right\}
$$

is closed and can be expressed as

$$
\left\{x \mid x \in D \quad f_{I}(x, \omega(x)) \geq a\right\}=\bigcup_{n=1}^{\infty}\left\{x \mid x \in D_{n} f_{I}(x, \omega(x)) \geq a\right\} .
$$

Thus, $\left\{x \mid x \in D_{n}, f_{I}(x, \omega(x)) \geq a\right\}$ is measurable, which implies that

$$
\left\{x \mid x \in D, f_{I}(x, \omega(x)) \geq a\right\}
$$

is measurable. Hence, $f_{I}(x, \omega(x))$ is measurable on $\Omega$. Then, $f(x, \omega(x))$ is measurable. This ends the proof.

In fact, this lemma is also true when mes $\Omega=+\infty$. For the Caratheodory operator $T$, we first prove that the operator maintains the convergence in measure for sequences of differential forms.

Theorem 3.1. Suppose that mes $\Omega<\infty$. If $\omega_{n}(s)(n=1,2, \ldots)$ converge in measure to $\omega(s)$ on $\Omega$, then $T \omega_{n}(s)=f\left(s, \omega_{n}(s)\right)(n=1,2, \ldots)$ converge in measure to $T \omega(s)$ on $\Omega$.

Proof.

For any $\sigma>0$, let $F_{n}=\left\{s|s \in \Omega| f,\left(s, \omega_{n}(s)\right)-f(s, \omega(s)) \mid \geq \sigma\right\}$. We need to prove

$$
\lim _{n \rightarrow \infty} \operatorname{mes} F_{n}=0 \text {, }
$$


that is

$$
\lim _{n \rightarrow \infty} \operatorname{mes} D_{n}=\operatorname{mes} \Omega,
$$

where

$$
D_{n}=\Omega \backslash F_{n}=\left\{s|s \in \Omega,| f\left(s, \omega_{n}(s)\right)-f(s, \omega(s)) \mid<\sigma\right\} .
$$

Let

$$
\begin{gathered}
\Omega_{k}=\left\{s \mid s \in \Omega \text { satisfy that for any } \omega^{\prime} ; \text { if }\left|\omega(s)-\omega^{\prime}\right|<\frac{1}{k}\right. \text {, then we have } \\
\left.\qquad\left|f(s, \omega(s))-f\left(s, \omega^{\prime}\right)\right|<\sigma\right\}(k=1,2, \ldots) .
\end{gathered}
$$

Clearly

$$
\Omega_{1} \subset \Omega_{2} \subset \Omega_{3} \subset \cdots .
$$

Let $H=\bigcup_{k=1}^{\infty} \Omega_{k}$. If $s_{0} \in \Omega \backslash H$, then $s_{0} \notin \Omega_{k}(k=1,2, \ldots)$. So there exists $\omega_{k}^{\prime}$ such that

$$
\left|\omega\left(s_{0}\right)-\omega_{k}^{\prime}\right|<\frac{1}{k} .
$$

But

$$
\left|f\left(s_{0}, \omega\left(s_{0}\right)\right)-f\left(s_{0}, \omega_{k}^{\prime}\right)\right| \geq \sigma \quad(k=1,2, \ldots) .
$$

Thus $f\left(s_{0}, \omega\right)$ is not continuous at $\omega=\omega_{0}=\omega\left(s_{0}\right)$. Hence, because of $f$ satisfying $C$ conditions, we know mes $(\Omega \backslash H)=0$, that is

$$
\lim _{k \rightarrow \infty} m e s \Omega_{k}=\text { mes } H=\operatorname{mes} \Omega .
$$

For all $\varepsilon>0$, we can choose sufficiently large $k_{0}$ such that

$$
m e s \Omega_{k_{0}}>\operatorname{mes} \Omega-\frac{\varepsilon}{2} \text {. }
$$

Let

$$
\begin{aligned}
& Q_{n}=\left\{s|s \in \Omega,| \omega_{n}(s)-\omega(s) \mid \geq \frac{1}{k_{0}}\right\} \\
& R_{n}=\Omega \backslash Q_{n}=\left\{s|s \in \Omega,| \omega_{n}(s)-\omega(s) \mid<\frac{1}{k_{0}}\right\} .
\end{aligned}
$$

As $\omega_{n}(s)$ converge in measure to $\omega(s)$, we have

$$
\lim _{n \rightarrow \infty} \operatorname{mes}_{n}=0 \text {, }
$$

that is

$$
\lim _{n \rightarrow \infty} \operatorname{mes}_{n}=\operatorname{mes} \Omega .
$$

Thus, there exists a positive integer $N$ such that

$$
m e s R_{n}>\operatorname{mes} \Omega-\frac{\varepsilon}{2} i f n>N .
$$


Obviously, $\Omega_{k_{0}} \cap R_{n} \subset D_{n}$, so

$$
\Omega \backslash D_{n} \subset \Omega \backslash\left(\Omega_{k_{0}} \cap R_{n}\right)=\left(\Omega \backslash \Omega_{k_{0}}\right) \cup\left(\Omega \backslash R_{n}\right) .
$$

Then, with (3.1) and (3.2), and mes $\Omega<\infty$, we know that for $n>N$,

$$
\begin{aligned}
0 & \leq \operatorname{mes} \Omega-\operatorname{mes}_{n}=\operatorname{mes}\left(\Omega \backslash D_{n}\right) \\
& \leq \operatorname{mes}\left(\Omega \backslash \Omega_{k_{0}}\right)+\operatorname{mes}\left(\Omega \backslash R_{n}\right) \\
& =\left(\operatorname{mes} \Omega-\operatorname{mes} \Omega_{k_{0}}\right)+\left(\operatorname{mes} \Omega-\operatorname{mes}_{n}\right) \\
& <\frac{\varepsilon}{2}+\frac{\varepsilon}{2}=\varepsilon .
\end{aligned}
$$

That is

$$
\lim _{n \rightarrow \infty} \operatorname{mes}_{n}=\operatorname{mes} \Omega,
$$

and we have

$$
\lim _{n \rightarrow \infty} \operatorname{mes} F_{n}=0
$$

Lemmas 3.3, 3.4 will be used in the following proof of the main theorem.

Lemma 3.3. In normed space, we have,

$$
\left.|| x|-| y\right|^{p} \leq\left.|| x\right|^{p}-|y|^{p} \mid
$$

where $x, y$ is any element of this space.

The proof is easy to obtain and therefore omitted.

Lemma 3.4. Suppose $\omega_{n}(s) \in L^{p}\left(\Omega, \Lambda^{l}\right)$ and $p \geq 1$. If $\left\|\omega_{n}-\omega_{0}\right\| \rightarrow 0$, then there exists a subsequence $\omega_{n_{k}}(s)$ of $\omega_{n}(s)$ such that $\omega_{n_{k}}(s) \rightarrow \omega_{0}(s)$ a.e.

Proof. For $\omega_{n}(s)=\Sigma_{I} \omega_{n, I}(s) d s_{I} \in L^{p}\left(\Omega, \Lambda^{l}\right)$, if

$$
\left\|\omega_{n}-\omega_{0}\right\|_{p} \rightarrow 0,
$$

using

$$
\left\|\omega_{n, I}-\omega_{0, I}\right\|_{p} \leq\left\|\omega_{n}-\omega_{0}\right\|_{p}=\left\|\left(\sum\left(\omega_{n, I}-\omega_{0, I}\right)^{2}\right)^{p / 2}\right\|_{p,}
$$

we have

$$
\left\|\omega_{n, I}-\omega_{0, I}\right\|_{p} \rightarrow 0, \text { for any } I .
$$

As we know, for the first index $I_{1}$, we have subsequence $\omega_{n_{k}, I_{1}}(s) \rightarrow \omega_{o, I_{1}}(s) a . e$.. And for $I_{2}$, we also have $\left\|\omega_{n, I_{2}}-\omega_{0, I_{2}}\right\| \rightarrow 0$. By fixing the index $I_{2}$, there exists a subsequence $\omega_{n_{k_{j}} I_{2}}(s)$ of the sequence $\omega_{n_{k}, I_{2}}$ satisfying $\omega_{n_{k_{j}}, I_{2}}(s) \rightarrow \omega_{0, I_{2}}(s)_{a . e .}$

By repeating the above procedure, we can find subsequence $\omega_{n, I}(s) \rightarrow \omega_{0, I}(s)$ a.e., for any $I$.

Hence, there exists a subsequence $\omega_{n, I}(s) \rightarrow \omega_{0, I}(s) a . e$. Thus, we complete the proof.

Theorem 3.2. Suppose mes $\Omega<\infty, p_{1}, p_{2} \geq 1$. If $f$ satisfies

$$
|f(s, \omega)| \leq a(s)+b|\omega|^{p_{1} / p_{2}} \quad s \in \Omega, \omega \in L^{p_{1}}\left(\Omega, \wedge^{\iota}\right),
$$

where $a(s) \in L^{p_{2}}(\Omega)$, and $b>0$ is a constant, then $C$-operator $T$ maps $L^{p_{1}}\left(\Omega, \wedge^{l}\right)$ into $L^{p_{2}}\left(\Omega, \wedge^{l}\right)$ and simultaneously is bounded and continuous.

Proof. If $\omega(s) \in L^{p_{1}}\left(\Omega, \wedge^{t}\right)$, we have $a(s)+b|\omega(s)|^{p_{1} / p_{2}} \in L^{p_{2}}(\Omega)$, which implies that $T \omega(s)=f(s, \omega(s)) \in L^{p_{2}}\left(\Omega, \wedge^{\imath}\right)$ and 


$$
T: L^{p_{1}}\left(\Omega, \wedge^{\imath}\right) \rightarrow L^{p_{2}}\left(\Omega, \wedge^{\imath}\right) .
$$

With Minkowski inequality, for any $\omega(s) \in L^{p_{1}}\left(\Omega, \wedge^{\imath}\right)$, we have

$$
\begin{aligned}
\|T \omega\|_{p_{2}} & \leq\left\|a(s)+b|\omega(s)|^{p_{1} / p_{2}}\right\|_{p_{2}} \\
& \leq\|a(s)\|_{p_{2}}+b\|\omega(s)\|_{p_{1}}^{p_{1} / p_{2}} .
\end{aligned}
$$

So $T$ is bounded. Next we prove the continuity of $T$.

If $T$ is discontinuous in $L^{p_{1}}\left(\Omega, \wedge^{\imath}\right)$, that is to say, there exist $\left\{\omega_{n}\right\} \subset L^{p_{1}}\left(\Omega, \wedge^{l}\right) \quad(n=0,1,2 \ldots)$ and $\varepsilon_{0}>0$, such that

$$
\left\|\omega_{n}(s)-\omega_{0}(s)\right\|_{p_{1}} \rightarrow 0,
$$

but

$$
\left\|T \omega_{n}(s)-T \omega_{0}(s)\right\|_{p_{2}} \geq \varepsilon_{0} .
$$

Let

$$
f_{n}(s)=T \omega_{n}(s)=f\left(s, \omega_{n}(s)\right), \quad g_{n}(s)=a(s)+b\left|\omega_{n}(s)\right|^{p_{1} / p_{2}} .
$$

Then

$$
\left|f_{n}(s)\right| \leq g_{n}(s) .
$$

According to Minkowski inequality, we have

$$
-\left\|\omega_{n}-\omega_{0}\right\|_{p_{1}} \leq\left\|\omega_{n}\right\|_{p_{1}}-\left\|\omega_{0}\right\|_{p_{1}} \leq\left\|\omega_{n}-\omega_{0}\right\|_{p_{1}}
$$

So

$$
\left\|\left|\omega_{n}\|-\| \omega_{0}\|\mid \leq\| \omega_{n}-\omega_{0} \|_{p_{1}} .\right.\right.
$$

and

$$
\left\|\omega_{n}-\omega_{0}\right\|_{p_{1}} \rightarrow 0,
$$

so

$$
\left\|\omega_{n}\right\|_{p_{1}} \rightarrow\left\|\omega_{0}\right\|_{p_{1}} .
$$

With the first one of $C$-conditions and Lemma 3.4, there exists a subsequence (suppose that $\left\{\omega_{n}\right\}$ is this subsequence) such that

$$
f_{n}(s) \rightarrow f_{0}(s), \quad g_{n}(s) \rightarrow g_{0}(s) \quad \text { a.e. }
$$

Obviously, $\left.|| \omega_{n}\right|^{p_{1}}-\left.\left|\omega_{0}\right|^{p_{1}}|\leq| \omega_{n}\right|^{p_{1}}+\left|\omega_{0}^{p_{1}}\right|$. According to Lemma Fatou, we have

$$
\begin{aligned}
& \int_{\Omega} \underline{\lim }_{n \rightarrow \infty}\left(\left|\omega_{n}\right|^{p_{1}}+\left|\omega_{0}\right|^{p_{1}}-\left.|| \omega_{n}\right|^{p_{1}}-\left|\omega_{0}\right|^{p_{1}} \mid\right) d s \\
& \leq \underline{\lim }_{n \rightarrow \infty} \int_{\Omega}\left(\left|\omega_{n}\right|^{p_{1}}+\left|\omega_{0}\right|^{p_{1}}-\left.|| \omega_{n}\right|^{p_{1}}-\left|\omega_{0}\right|^{p_{1}} \mid\right) d s .
\end{aligned}
$$

Then

$$
2\left\|\omega_{0}\right\|_{p_{1}}^{p_{1}} \leq 2\left\|\omega_{0}\right\|_{p_{1}}^{p_{1}}-\underline{\lim }_{n \rightarrow \infty} \int_{\Omega}\left(\left.|| \omega_{n}\right|^{p_{1}}-\left|\omega_{0}\right|^{p_{1}} \mid\right) d s .
$$


Hence, there exists a subsequence $\left\{\omega_{n_{k}}(s)\right\}$ such that

$$
\left.\lim _{k \rightarrow \infty} \int_{\Omega}|| \omega_{n_{k}}\right|^{p_{1}}-\left|\omega_{0}\right|^{p_{1}} \mid d s=0
$$

Suppose that $\left.\lim _{n \rightarrow \infty} \int_{\Omega}|| \omega_{n}\right|^{p_{1}}-\left|\omega_{0}\right|^{p_{1}} \mid d s=0$

and with Lemma 3.3, we have

$$
\begin{aligned}
\int_{\Omega}\left|g_{n}(s)-g_{0}(s)\right|^{p_{2}} d s & =b^{p_{2}} \int_{\Omega} \|\left.\omega_{n}\right|^{p_{1} / p_{2}}-\left.\left|\omega_{0}\right|^{p_{1} / p_{2}}\right|^{p_{2}} d s \\
& \leq b^{p_{2}} \int_{\Omega}\left\|\left.\omega_{n}\right|^{p_{1}}-\left|\omega_{0}\right|^{p_{1}}\right\| d s \rightarrow 0 \quad(n \rightarrow \infty) .
\end{aligned}
$$

As

$$
\left.\lim _{n \rightarrow \infty} \int_{\Omega}|| \omega_{n}\right|^{p_{1}}-\left|\omega_{0}\right|^{p_{1}} \mid d s=0,
$$

we have

$$
\lim _{n \rightarrow \infty} \int_{\Omega}\left|g_{n}(s)-g_{0}(s)\right|^{p_{2}} d s=0 .
$$

Then

$$
\left\|g_{n}(s)\right\|_{p_{2}} \rightarrow\left\|g_{0}(s)\right\|_{p_{2}} \quad(n \rightarrow \infty) .
$$

And

$$
\left|f_{n}(s)-f_{0}(s)\right|^{p_{2}} \leq 2^{p_{2}}\left(\left|g_{n}(s)\right|^{p_{2}}+\left|g_{0}(s)\right|^{p_{2}}\right) .
$$

Applying Lemma Fatou to $\left\{2^{p_{2}}\left|g_{n}(s)\right|^{p_{2}}+\left|g_{0}(s)\right|^{p_{2}}-\left|f_{n}(s)-f_{0}(s)\right|^{p_{2}}\right\}$, we have

$$
\underline{\lim }_{n \rightarrow \infty} \int_{\Omega}\left|f_{n}(s)-f_{0}(s)\right|^{p_{2}} d s=0 .
$$

According to Lemma 3.4, there exists a subsequence $\left\{f_{n_{k}}\right\}$ such that

$$
\lim _{k \rightarrow \infty} \int_{\Omega}\left|f_{n_{k}}(s)-f_{0}(s)\right|^{p_{2}} d s=\underline{\lim }_{n \rightarrow \infty} \int_{\Omega}\left|f_{n}(s)-f_{0}(s)\right|^{p_{2}} d s=0,
$$

that is

$$
\left\|T \omega_{n_{k}}-T \omega_{0}\right\| \rightarrow 0 \quad(k \rightarrow \infty) .
$$

This is contradictory to $\left\|T \omega_{n}(s)-T \omega_{0}(s)\right\|_{p_{2}} \geq \varepsilon_{0}$. Hence, $T$ is continuous. This ends the proof.

Actually, we can prove that the condition in Theorem 3.2 is a necessary and sufficient condition.

Theorem 3.3. The Caratheodory operator $T$ maps continuously and boundedly $L^{p_{1}}\left(\Omega, \wedge^{\imath}\right)$ into $L^{p_{2}}\left(\Omega, \wedge^{\imath}\right)$, if and only if, there exists $b>0, a(x) \geq 0, a(x) \in L^{p_{2}}(\Omega)$ satisfying the following inequality,

$$
|f(x, \omega)| \leq a(x)+b|\omega|^{\frac{p_{1}}{p_{2}}} \quad\left(x \in \Omega, \omega \in \wedge^{\imath}\right) .
$$

Proof. The proof of sufficiency has been proved in Theorem 3.2. 
Proof of necessity. First, we suppose $f(x, 0) \equiv 0$. With the continuity and boundedness of $T$, we know there exists $b>0$ such that

$$
\int_{\Omega}|\omega(x)|^{p_{1}} d x \leq 1 \Rightarrow \int_{\Omega}|f(x, \omega(x))|^{p_{2}} d x \leq b^{p_{2}} .
$$

Then, we define a function on $\Omega \times \wedge^{l}$ :

$$
g(x, \omega)= \begin{cases}|f(x, \omega)|-b|\omega|^{\frac{p_{1}}{p_{2}}}, & \text { if }|f(x, \omega)| \geq b|\omega|^{\frac{p_{1}}{p_{2}}} \\ 0, & \operatorname{if}|f(x, \omega)| \geq b|\omega|^{\frac{p_{1}}{p_{2}}} .\end{cases}
$$

Suppose that $\omega(x) \in L^{p_{1}}\left(\Omega, \wedge^{\prime}\right)$. Let $F=\{x \mid x \in \Omega, g(x, \omega(x))>0\}$ and $\int_{F}|\omega(x)|^{p_{1}} d x=n+\alpha$, where $n$ is a nonnegative integer and $0 \leq \alpha<1$. With the absolute continuity of integral, we can divide $F$ into $n+1$ measurable set $\Omega_{1}, \Omega_{2}, \ldots, \Omega_{n+1}$ which is mutually disjoint and

$$
\int_{\Omega_{i}}|\omega(x)|^{p_{1}} d x \leq 1 \quad(i=1,2, \ldots, n+1) .
$$

Hence, according to (3.4), we have

$$
\begin{aligned}
\int_{F}|f(x, \omega(x))|^{p_{2}} d x & =\sum_{i=1}^{n+1} \int_{\Omega_{i}}|f(x, \omega(x))|^{p_{2}} d x \\
& \leq(n+1)^{b^{p_{2}}}
\end{aligned}
$$

Then

$$
\begin{aligned}
\int_{\Omega}[g(x, \omega(x))]^{p_{2}} d x & =\int_{F}[g(x, \omega(x))]^{p_{2}} d x \\
& =\int_{F}\left[\left.f(x, \omega(x))|-b| \omega(x)\right|^{\frac{p_{1}}{p_{2}}}\right] d x \\
& \leq \int_{F}\left[\left.f(x, \omega(x))\right|^{p_{2}} d x-b^{p_{2}} \int_{F}|\omega(x)|^{p_{1}} d x\right. \\
& \leq(n+1) b^{p_{2}}-(n+\alpha)^{b_{2}} \\
& \leq b^{p_{2}} .
\end{aligned}
$$

We use the following inequality in the above statement,

$$
(u-v)^{r} \leq u^{r}-v^{r}, \quad \forall u \geq v \geq 0, r \geq 1 .
$$

As $f(x, \omega)$ satisfies the Caratheodory condition, $g(x, \omega)$ also satisfies it. Then there exist $D \subset \Omega$, mes $(\Omega \backslash D)=0$, and $f(x, \omega)$ is continuous with respect to $\omega$ for any $x \in D$. Let $D=\bigcup_{k=1}^{\infty} D_{k}$, where $m e s D_{k}<+\infty(k=1,2, \ldots)$ and D1 $\subset$ D2 $\subset$ D3 $\subset \ldots$. Let $\omega_{k}(x)=$ $\Sigma_{I} \omega_{k, I}(x) d x_{I}$ on $x \in D_{k}$, where for any $I$,

$$
-k \leq \omega_{I}^{*} \leq k, \text { and } g(x, \omega *)=\max _{-k \leq \omega_{I} \leq k} g(x, \omega) .
$$

We choose $\omega_{k}$ as follows. First, we sort all of the $I$ with an order $\omega_{1}, \omega_{2}, \ldots, \omega_{r}\left(r \triangleq C_{N}^{l}\right)$. For $I_{1}$, let $\omega_{I_{1}}^{*}$ be the smallest value of which satisfies the above qualification; for $I_{2}$, let $\omega_{I_{2}}^{*}$ be the smallest value of which satisfies the above qualification and under the condition that we have selected $\omega_{I_{1}}^{*}$; then we repeat this procedure. 
Hence, $\omega_{k}(x)=\sum_{I} \omega_{k, I}(x) d x_{I}=\sum_{I} \omega_{I}^{*} d x_{I}$ on $D_{k}$. If $x \in \Omega \backslash D_{k}$, let $\omega_{k, I}=0$, then $\omega_{k}(x)=$ 0 .

Now we prove that $\omega_{k, I}(x)$ is measurable on $\Omega$ for any $I$. Obviously we only need to prove this on $D_{k}$. We suppose that any other $\omega_{k, I}$ is fixed where the maximum can be got; then $g(x, \omega)$ only has two variables $x, \omega_{I}$. Hence, let $g\left(x, \omega_{I}\right)$ stand for the function we are studying here, which actually is $g(x, \omega), \omega=\sum_{I}, \omega_{I}^{*}, d x_{I}, d x_{I},+\omega_{k, I} d x_{I}$. Using Lemma (3.1), there exists bounded closed set $F_{k, n} \subset D_{k}, m e s F_{k, n}>\operatorname{mes} D_{k}-\frac{1}{n} \quad(n=1,2, \ldots)$ such that $g\left(x, \omega_{I}\right)$ is continuous on $F_{k, n}$ $\times(-\infty,+\infty)$. Clearly, we can suppose $F_{k, n} \subset F_{k, n+1}(n=1,2, \ldots)$. Let $G_{k}=\bigcup_{n=1}^{\infty} F_{k, n}$. Then mes $\left(D_{k} \backslash G_{k}\right)=0$. For any real number $a$, we investigate the set $H_{k, n}=\left\{x \mid x \in F_{k}\right.$, $\left.{ }_{n}, \omega_{k, I}>a\right\}$. Suppose that $x_{0} \in H_{k, n}$, let $\eta=\omega_{k, I}\left(x_{0}\right)-a>0$, and we select $\delta>0$ such that $\delta<\eta$ and $-k<\omega_{k, I}-\delta$. According to the definition of $\omega_{k, I}$, we know $g\left(x_{0}, \omega_{I}\right)<g\left(x_{0}\right.$, $\left.\omega_{k, I}\left(x_{0}\right)\right)$, for all $-k \leq \omega<\omega_{k, I}\left(x_{0}\right)$. Hence,

$$
2 \delta=g\left(x_{0}, \omega_{k, I}\left(x_{0}\right)\right)-\max _{-k \leq \omega \leq \omega_{k, I}\left(x_{0}\right)-\delta} g\left(x_{0}, \omega_{I}\right)>0 .
$$

With the uniform continuity of $g\left(x, \omega_{I}\right)$ on $F_{k, n} \times[-k, k]$, there exists $\rho_{x 0}>0$ such that if $\left|x-x_{0}\right|<\rho_{x_{0}}$ and $x \in F_{k, n}$, then we always have

$$
\left|g\left(x, \omega_{I}\right)-g\left(x_{0}, \omega_{I}\right)\right|<\delta \text { for all }-k \leq \omega \leq k .
$$

Hence, we know that

$$
g\left(x, \omega_{k, I}\left(x_{0}\right)\right)>g\left(x, \omega_{I}\right), \text { for all }-k \leq \omega_{I} \leq \omega_{k, I}\left(x_{0}\right)-\delta .
$$

Then $\omega_{k, I}(x)>\omega_{k, I}\left(x_{0}\right)-\delta>\omega_{k, I}\left(x_{0}\right)-\eta=a$, for all $\left|x-x_{0}\right|<\rho_{x_{0}}, x \in F_{k, n}$. Let $S\left(s_{0}, \rho_{x_{0}}\right)$ stand for the open ball $\left\{x\left|x \in R^{N},\right| x-x_{0} \mid<\rho_{x_{0}}\right\}$. Then we have proved $S\left(x_{0}, \rho_{x_{0}}\right) \cap F_{k, n} \subset H_{k, n}$. Hence, we know that

$$
H_{k, n}=\left(\bigcup_{x \in H_{k, n}} S\left(x, \rho_{x}\right)\right) \cap F_{k, n} .
$$

As $\bigcup_{x \in H_{k, n}} S\left(x, \rho_{x}\right)$ is open set and $F_{k, n}$ is closed set, $H_{k, n}$ is measurable. Thus, $\left\{x \mid x \in G_{k}, \omega_{k, I}>a\right\}=\bigcup_{n=1}^{\infty} H_{k, n}$ is also measurable. Then we know that $\omega_{k, I}(x)$ is measurable on $G_{k}$, so is on $D_{k}$.

Now we prove that $\omega_{k, I}(x)$ is measurable for any $I$. According to Lemma 3.2, $f\left(x, \omega_{k}\right.$ $(x))$ is measurable, therefore $g\left(x, \omega_{k}(x)\right)$ is measurable, too.

Obviously,

$$
\int_{\Omega}|\omega(x)|^{p_{1}} d x=\int_{D_{k}}\left|\omega_{k}(x)\right|^{p_{1}} d x \leq\left(C_{n}^{l}\right)^{p_{1}} k^{p_{1}} \operatorname{mes} D_{k}<+\infty .
$$

So $\omega_{k}(x) \in L^{p_{1}}\left(\Omega, \wedge^{\iota}\right)$. Using (3.5), we have

$$
\int_{\Omega}\left[g\left(x, \omega_{k}(x)\right)\right]^{p_{2}} d x \leq b^{p_{2}} \quad(k=1,2, \ldots) .
$$

Let $a(x)=\sup _{\omega \in \wedge^{\prime} g}(x, \omega) \quad(x \in \Omega)$.. 
Clearly, when $x \in D$, we have

$$
a(x)=\lim _{k \rightarrow \infty} g\left(x, \omega_{k}(x)\right),
$$

and so $a(x)$ is nonnegative measurable function. With (3.6) and (3.7), using Lemma Fatou, we have that

$$
\int_{\Omega}[a(x)]^{p_{2}} d x \leq \underline{\lim }_{k \rightarrow \infty} \int_{\Omega}\left[g\left(x, \omega_{k}(x)\right)\right]^{p_{2}} d x \leq b^{p_{2}} .
$$

Hence, $a(x) \in L^{p_{2}}(\Omega)$. As

$$
a(x)=\sup _{\omega \in \wedge^{\prime} g}(x, \omega) \geq \sup _{\omega \in \wedge^{\iota}}\left\{|f(x, \omega)|-b|\omega|^{\frac{p_{1}}{p_{2}}}\right\},
$$

thus

$$
|f(x, \omega)| \leq a(x)+b|\omega|^{\frac{p_{1}}{p_{2}}} \quad\left(x \in \Omega, \omega \in \wedge^{l}\right) .
$$

Hence, under the hypothesis $f(x, 0) \equiv 0$, we prove the necessity of the theorem. For general situation, let $f_{1}(x, \omega)=f(x, \omega)-f(x, 0)$. Then $f_{1}(x, 0) \equiv 0$. Applying the above conclusion to $f_{1}$, we know that there exist $a_{1}(x) \geq 0, a_{1}(x) \in L^{p_{1}}(\Omega)$ and $b>0$, such that

$$
\left|f_{1}(x, \omega)\right| \leq a(x)+b|\omega|^{\frac{p_{1}}{p_{2}}}, \quad \forall x \in \Omega, \quad \omega \in \wedge^{\prime},
$$

where $a(x)=a_{1}(x)+|f(x, 0)| \geq 0, a(x) \in L^{p_{2}}(\Omega)$. Then we know $f(x, \omega) \in L^{p_{2}}\left(\Omega, \wedge^{l}\right)$. Hence, we complete the proof.

In the proofs above, when $l=0$, Caratheodory operator $T$ : $\Omega \times R \mapsto \Omega \times R$ degenerates to normal function space. We can also find that when $l_{1} \neq l_{2}$, these conclusions are still true. Actually, we only need to redefine an appropriate norm. For $\omega(x)=\sum_{l=0}^{n} \omega_{l}(x) \in \wedge$, let

$$
\|\omega(x)\|_{\Omega, \wedge, p}=\sum_{l=0}^{n}\left\|\omega_{l}(x)\right\|_{\Omega, \wedge^{\prime}, p}
$$

where $\omega_{l}(x)=\Sigma_{I} \omega_{I, l}(x) d x_{I}$. Then we can extend $T$ to $L^{p_{1}}(\Omega \times \wedge) \mapsto L^{p_{2}}(\Omega \times \wedge)$ and the above conclusions still come into existence.

\section{Acknowledgements}

ZT was supported by the Fundamental Research Foundation of NUDT (NO. JC10-02-02), and by the NSF of Hunan Province (No. 11JJ3004).

\section{Authors' contributions}

$\mathrm{JZ}$ carries out the main idea and ZT gives the main proof.

\section{Competing interests}

The authors declare that they have no competing interests.

Received: 15 May 2011 Accepted: 18 October 2011 Published: 18 October 2011

\section{References}

1. Agarwal, PR, Ding, S, Nolder, CA: Inequalities for Differential Forms. Springer (2009)

2. Cao, Z, Bao, G, Xing, Y, Li, R: Some Caccioppoli estimates for differential forms. J Inequal Appl 2009, 11 (2009). Article ID 734528 ,

3. Aronsson, G, Lindqvist, P: On p-harmonic functions in the plane and their stream functions. J Diff Eqns. 74, 157-178 (1988). doi:10.1016/0022-0396(88)90022-8 
4. Ball, JM: Convexity conditions and existence theorems in nonlinear elasticity. Arch Rational Mech Anal. 63, 337-403 (1977)

5. Ding, S: Two-weight Caccioppoli inequalities for solutions of nonhomogeneous A-harmonic equations on Riemannian manifolds. Proc Am Math Soc. 132, 2367-2375 (2004). doi:10.1090/S0002-9939-04-07347-2

6. Ding, S: Local and global norm comparison theorems for solutions to the nonho-mogeneous A-harmonic equation. J Math Anal Appl. 335, 1274-1293 (2007). doi:10.1016/j.jmaa.2007.02.048

7. Giaquinta, M, Soucek, J: Caccioppoli's inequality and Legendre-Hadamard condition. Math Ann. 270, 105-107 (1985). doi:10.1007/BF01455535

8. Iwaniec, T, Sbordone, G: Weak minima of variational integrals. J Reine Angew Math. 454, 143-161 (1994)

9. Nolder, CA: Hardy-Littlewood theorems for A-harmonic tensors. Illinois J Math. 43, 613-631 (1999)

10. Nolder, CA: Global integrability theorems for A-Harmonic tensors. J Math Anal Appl. 247, 236-247 (2000). doi:10.1006/ jmaa.2000.6850

11. Nolder, CA: Conjugate harmonic functions and Clifford algebras. J Math Anal Appl. 302, 137-142 (2005). doi:10.1016/j. jmaa.2004.08.008

12. Stroffolini, B: On weakly A-harmonic tensors. Stud Math. 114, 289-301 (1995)

13. Dajun, G: Nonlinear Functional Analysis. Shandong Science and Technology Press (2001)

14. Zhaoyong, Y, Huaiyun, G, Zongben, X: Nonlinear Analysis. Xi'an Jiaotong University Press (1991)

15. Longguang, $\mathrm{H}$ : The relationship between closed functional and continuous functional. J Longyan Teachers Coll (Sci Nat Ed). 8, 10-15 (1990)

doi:10.1186/1029-242X-2011-88

Cite this article as: Tang and Zhu: Caratheodory operator of differential forms. Journal of Inequalities and Applications 2011 2011:88.

\section{Submit your manuscript to a SpringerOpen ${ }^{\circ}$} journal and benefit from:

- Convenient online submission

- Rigorous peer review

- Immediate publication on acceptance

- Open access: articles freely available online

- High visibility within the field

- Retaining the copyright to your article

Submit your next manuscript at $\boldsymbol{s p r i n g e r o p e n . c o m ~}$ 